\title{
The Effect of music on maternal anxiety and maternal and fetal heart rate during cardiotocography
}

\section{Gabriella Milona, Vasilios Pergialiotis, Marianna Theodora, Dimitrios Loutradis, Georgios Daskalakis}

Alexandra Hospital, National and Kapodistrian University of Athens, Greece

Corresponding Author

Gabriella Milona MSc, e-mail: gabriellamilona@hotmail.com

\section{Abstract}

Introduction: It is well known that music acts as an analgesic. Facilitates muscle relaxation, reduction of physical pain and mental tension.

Purpose of this original investigation: study the effect of music on maternal anxiety during cardiotocography as well as its effect on embryonic cardiac function in relation to mother's heart rate.

Method and materials: The survey, conducted in external midwifery medical services of Alexandra's Hospital, involved 80 pregnant women who met the criteria for participation. They were divided in two groups. The 40 pregnant women were the Musical Group, and the other 40 were the Control Group. In both groups, the STAI scale was used. The control group underwent cardiotocography examination without listening to music. The Musical Group were selected to hear the music track 'Kung Fu Piano: Cello Ascends', a cover of the Piano Guys band. The hearing started 5 minutes before the end of the cardiotocography, with headset playing frequencies that are within the frequency spectrum of the music track and special music player, and 10 minutes before the end of the cardiotocography the pulse oximeter Beurer P080 was placed. In this music track we did music and frequency analysis with the following programs: SPAN of Voxeno and Reaper of Cocos, as well as Theory-Harmony of Music, to see if at the time certain changes in the track occur, there are corresponding changes in the heart rate of the mother and the fetus.

Results: In our study we observed that music significantly decreased the level of anxiety of women subjected to non-stress test (NST) (Median anxiety score prior to the conduct of the non-stress test 53.38 (49-57) vs 25.20 (23-28) following the completion of the test. Moreover differences among women that heard music were significant compared to those that did not (25.20 (21-28) vs 56 (48-64)) despite the fact that baseline differences among the two groups were comparable (54.45 (59-67) vs 50.80 (53-58). Finally, following performance of music analysis we observed significant variations in the baseline heartbeat of pregnant women as well as in the cardiotocographic analysis of fetuses (number of accelerations, baseline rhythm); those patterns were directly related to musical characteristics of the track that women listened to. 
Conclusions: Music has a positive effect on pregnancy. It is a non-invasive way of anxiety relief, as well as a simple, non-time-consuming way of improving cardiotocography among low risk cases; thus, potentially diminishing false-positive results which may result in unnecessary deliveries.

Key words: Music, pregnancy, non stress test, cardiotocography, STAI-Y scale, music analysis

\section{Introduction}

Cardiotocography is frequently performed to assess fetal wellbeing and is considered a significant aspect of the fetal biophysical profile assessment ${ }^{1}$. Proper interpretation of cardiotocography is vital in deciding the outcome of childbirth; however, the test per se is accompanied by significant false positive results; therefore, increasing the rates of unnecessary inductions of labor and cesarean sections ${ }^{2}$.

The MIDIRS centre showed that electronic monitoring alone increased the number of invasive births by $30 \%{ }^{3}$. Due to the increased level of intervention associated with electronic monitoring, $\mathrm{NICE}^{4}$ and MIDIRS $^{3}$ recommended that the method of choice for the normal childbirth is the intermittent hearing.

Music is increasingly being used to treat cardiovascular, neurological and respiratory diseases ${ }^{5}$. A large majority of clinical studies dealing with the effects of music on various diseases related to the neurological piece such as Parkinson's, Alzheimer's, multiple sclerosis, etc. showed positive results of music as to the outcome of diseases ${ }^{6-9}$. Researches have also shown that listening to music can affect heart and respiratory rhythm as well as blood pressure $^{5,6}$ In addition, previous research reveals that listening to music is a non-intrusive way of analgesia and stress relief ${ }^{10-15}$.

Finally, researches show that listening to music has a positive effect not only on people suffering from a disease but also on pregnant women in pregnancy and childbirth ${ }^{10,16-20}$.

In the present study we sought to evaluate the potential beneficial effect of music hearing during the non-stress test on the psychological state of pregnant women. Furthermore, this research want to take into account the fetal cardiac function with the heart rate of the mother who heard music at the time of the cardiotocography and investigate the impact of music on the rates of the false positive results.

Moreover, the aim is to prove that music can have positive effects not only on the pregnant woman and on the fetus but also on health professionals as it can be a simple non-time-consuming way to try to improve cardiotocography in case of false negative cardiotocographys which will result in reducing unnecessarily invasive births.

\section{Materials and Methods}

The research was carried out in Athens at the G.N.A Alexandra. The study was approved by the Scientific Council of the General Staff of Alexandra (Application Number 743/14-09-2018) and was designed in accordance with the Declaration of Helsinki concerning Human Rights. All participants in the survey were properly informed and signed an informed consent before they were considered as eligible for inclusion.

Singleton pregnant women at term that referred to the outpatient obstetrical department of our hospital were selected. In order to be able to participate in the research they had to be over 18 years old, to know Greek or English language well, to be for the first time in pregnancy, neither to be now nor to have ever been existed users of addictive substances and not to take drugs that act in the central nervous system. The exclusion criteria included multiple 
pregnancies, preterm pregnancies and any pathology in pregnancy.

The pregnant women were separated in two groups. Only 40 women listened to music and those belonged to the Music Group while the other 40 belonged to the Control Group. The research was separated in two parts. In both places the psychology of the pregnant woman was assessed using the Spielberger State-Trait Anxiety Inventory (STAI) Transitional Anxiety Assessment Scale (STAI).

In the first part of this research which concerns the psychological piece, we asked from the 40 women that comprised the control group to complete the first 20 questions on the STAI scale that refer to the emotional state of the person generally, that is the permanent anxiety as a feature of personality. Then we subjected them to a non-stress test for about 20 to $25 \mathrm{~min}$ and at the end of this process women were asked to complete the remaining 20 questions on the STAI scale that refer to the emotional state of the examinee at the time of completion of the questionnaire, that is the transient anxiety as a result of the present situation.

The 40 women that comprised the Music Group also answered the first 20 questions on the STAI scale that refer to permanent anxiety and were subjected to a non-stress test. At 8 minutes we asked the remaining 20 questions on the STAI scale refer to transient stress while at the same time the cardiotocography continued to record. Ten minutes before the end of the cardiotocography we placed the pulse oximeter which we initially let record for 5 minutes so that we ensured that the measurements had stabilized and in the last 5 minutes we started the listening of a particular music track via an audio device with headphones, in which we have done music analysis. At the end of the music listening process women were disconnected from the cardiotocography device and completed the 20 questions on the STAI scale that refer to transient anxiety, that is the emotional state of the examinee at the time of completion of the questionnaire.
In the second part of the research, fetal heart function was taken into account with the mother's heartbeat. Initially a music analysis was performed on the music track we selected and then the changes that occur in the heart rate of the fetus and the heart rate of the mother were observed at the very moment when certain changes occur in the piece. For the music analysis, two programs on the computer were used as well as knowledge from Music Theory to see if at the time changes occur in the track there are corresponding changes in the heart rate of the mother and the fetus.

The programs that we used are Voxeno's Sound Spectrum Analyzer (SPAN) and Cocos's Reaper owned by Digital Audio Workstation (DAW, digital audio editor). In Reaper we put Voxeno's SPAN which is a program that plays only through DAW and which is Spectrum Analyzer (spectral sound analyzer). However, without the knowledge given to us by the 'Theory of Music' (musical notes, note values, rhythmic education, musical intervals, scales, chords, musical expression and musical notation) it could not become a complete Music Analysis.

The music was chosen to be listened with headphones that play frequencies within the frequent spectrum of the piece and allow changes in ear pads to properly protect the hygiene of the pregnant and with a special music player so that it is safe to remain close to the pregnant woman but also not to creates interference that prevents the conduct of examinations. Furthermore, the headphones were not placed directly in the abdomen of the pregnant, because this is based on international protocols which said that this is not considered safe.

Continuous variables are presented as median (range) values. Friedman`s test was used to compare median values between groups due to their non-normal distribution. All reported analyses were designed as two-tailed. Statistical significance was set at $\mathrm{p}<0.05$. The SPSS statistical package was used 
in this study (IBM Corp. Released 2013. IBM SPSS Statistics for Windows, Version 22.0. Armonk, NY: IBM Corp.).

\section{Results}

Baseline characteristics of pregnant women were comparable among the two groups in terms of anxiety as depicted in Table 1. Significant differences were observed in anxiety scores among women that heard music, compared to those that did not (following completion of the cardiotocography). The decline in anxiety scores was statistically significant in the music group; whereas women that did not hear music had slightly higher (but non-significant) anxiety scores following completion of the test. Significant differences were also observed in anxiety scores to the music group before and after listening to music. The decline in anxiety scores was statistically significant after listening to music; whereas anxiety before the listening of music was significantly higher.

Some of the frequency values of the track are presented in Figure 1.

Qualitative analysis of maternal heartbeat patterns revealed variability of beats per minute that was directly related to the frequency values of the musical piece. Furthermore, fetal response was documented in the form of accelerations and increase of baseline activity.
Table 1. Maternal anxiety scores

\begin{tabular}{lccc}
\hline & MUSIC GROUP & CONTROL GROUP & P-VALUE \\
Pre-test & $53(49-57)$ & $53(45-58)$ & .829 \\
Post-test & $23(21-28)$ & $59.5(43-67)$ & $<.001$ \\
p-value & $<.001$ & .08 &
\end{tabular}

\section{Conclusions}

From the first part of the research there was a statistically significant difference between the transient anxiety of the Control Group and the transient anxiety of the Music Group after listening to music. The group that listened to music during the cardiotocography showed after the end of the hearing, much lower levels of anxiety than the group that did not listen to music. Also, to the pregnant women belonging to the Music Group found a statistically significant difference in transient anxiety before listening to music and transient anxiety after listening to music. After the listening of the music track, pregnant women showed lower levels of anxiety. In addition, no statistically significant difference was found between the permanent stress of the Music Group and the permanent stress of the Control Group. This means that in both groups the stress was generally the same before the cardiotocography procedure began.

From the second part of the research which concern taking into account of fetal cardiac function

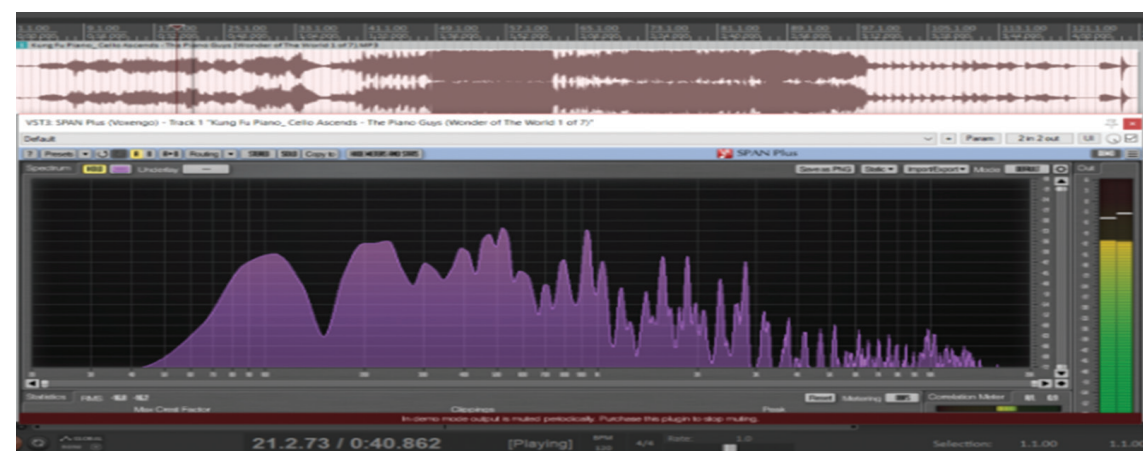

Figure 1. Some of the Frequency values of the track 


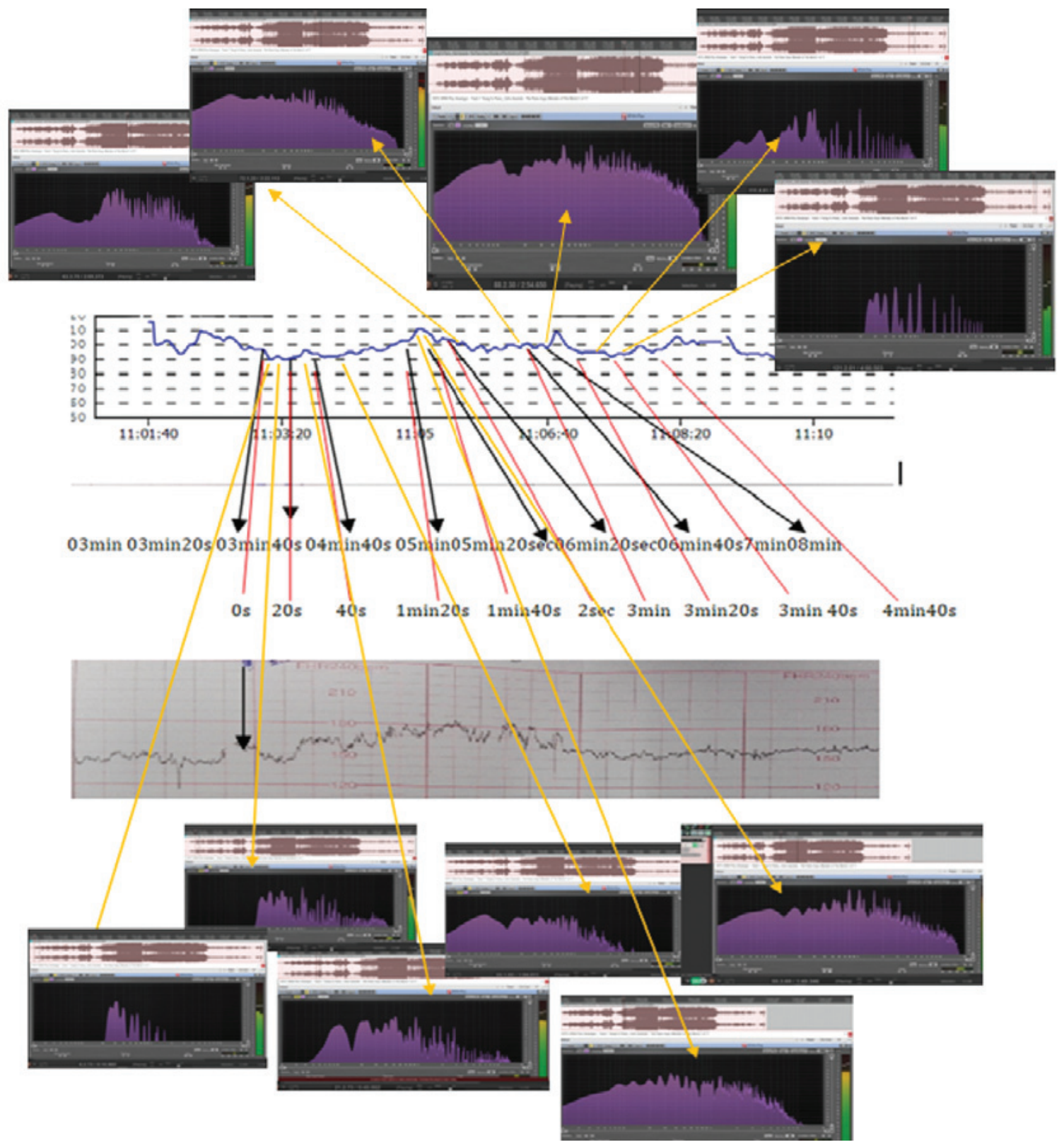

Figure 2. Example A: Values of pulse oximeter -Cardiotocography - some of the frequency values of the track.

with the heart function of the mother, the time of the cardiotocography and of the hearing of the "Kung Fu Piano-Cello ascends" (which had been preceded by music analysis by us), shows that the heartbeat of the pregnant women who belonged to the Music
Group, as well as of their fetus, are directly affected by music. Depending on the frequencies, decibels, dynamics, music values, tempo that exist in every second the heartbeat of both pregnant women and embryos exhibit specific changes that are perfectly 
related to each other and perfectly match the changes in the piece that we identify and study through the music analysis we have made.

Finally it was found that the music greatly improved the cardiotocographys. After the start of listening to music there is an increase in the baseline from $10 \mathrm{~b} / \mathrm{m}$ to $25 \mathrm{~b} / \mathrm{m}$, the appearance or increase of the number of accelerations and embryonic movements as well as an increase in variability from $5-10 \mathrm{~b} / \mathrm{m}$ to $10-15 \mathrm{~b} / \mathrm{m}$.

In our previous research in 2015 on 'Study of the effect of Music on mother and fetus in relation to maternal psychology and cardiotocography in pregnancy and childbirth some of the results of this research come to agree with this research such as the increase observed in variability from $5-10 \mathrm{~b} / \mathrm{m}$ to $15-20 \mathrm{~b} / \mathrm{m}$, the increase in the baseline, the increase in the number of accelerations and the increase in fetal movements observed in the cardiotocographys and to the group of pregnant women who listened to music of their own choice as well as to the group of pregnant women who listened to a particular piece in which we had done music analysis ${ }^{10}$.

of course, this research came to go a step further the 2015 survey after the add of pulse oximeter was used to monitor the mother's heart rate as well as two professional audio programs (on the computer) which come to create a more complete music analysis that will lead us to clearer conclusions. In terms of stress, that survey of 2015 found no statistically significant difference in temporary anxiety levels, although there was clearly a slight increase in the level of anxiety in the Control group. In the present survey, however, there was a statistically large difference in stress levels between the Music Group and the Control Group, with the Control group showing very high levels of anxiety compared to the group listening to music.

In another research we found some common results with our own research such as that lower scores of stress were observed to the group who listened to music compared to the control group as well as the frequency of acceleration in the heart rate of fetus increased significantly in the experimental group compared to the control group. Of course, in that research is stated that the basic fetal heartbeat was lower in the group listening to music, which did not occur in this research, on the contrary we observed an increase in basic fetal heart rate in the Music Group ${ }^{21}$.

In addition, in accordance with our results, two studies also reported that the non stress test examination causes stress to the pregnant women and listening to the music during the examination, has a positive effect on the embryonic parameters considered $^{22,23}$.

This research is a groundbreaking study. There are surveys that have combined music with PregnancyChildbirth, but there is no research similar to this study. In this research, detailed music analysis was performed on each msec of the piece we selected using two professional audio programs combined with knowledge from Music Theory. Then we studied one by one the cardiotocographys as well as the pdf of the pulse oximeter from each woman and in the end for each woman we compared in each sec-msec the cardiotocography, the result of the pulse oximeter and what happens in the music piece at that time with the help of music analysis trying to explain why we have the specific effects on the fetal pulses and the pulses of the woman.

Other advantages of this research are that we observed time savings in the examination of the cardiotocography and that no other interference, such as a change of position, giving juice, sweet or frozen water was deemed necessary in cases where the fetus was not kinetic. In the Music Group those embryos that were not kinetic at the beginning of the examination, after listening to music were activated and the cardiotocography was normal, exclusively 
using music. So in this way at the same time we gained time and it did not take the pregnant women whose embryos were less kinetic to go for a walk and to eat something sweet to activate the fetus. They finished their examination on time while at the same time this was positive for the staff of the Maternity Hospital as the cardiotocographys of the Music Group finished in the already calculated time that corresponds to them without having to re-examine them due to a non-reassuring cardiotocography.

Moreover, it is extremely important that with this research, we have also seen a great improvement in the interpersonal relations. Pregnant women, who listened to music, as we saw from our statistical results, were noticeably calmer with noticeably less anxiety than pregnant women who did not listen to music. This made them more cooperative with the staff, so the communication between them and the communication between the staff is clearly healthier. The additional advantage is that by listening to Music those falsely unreassuring cardiotocographys improved and we avoided additional interventions that one of them could be the hasty Caesarean Section. Another advantage is that music does not have the ability to improve the pathological cardiotocographys and this is an advantage because we could not use it safely in pregnant women if it hided the embryos that showed really non-reassuring cardiotocographys.

But in every study except the advantages, we also have the disadvantages. One drawback of this research is that it was done in the context of the Master's degree, so the time was limited. The second disadvantage is that our research was done in a small sample of the population (80 pregnant women) because many pregnant women who came to the outpatient clinics of G.N.A. Alexandra for the examination of the cardiotocography did not meet the criteria for participation in the research. For example, there were many women who were not their first baby, others who could not understand Greek or English also came several pregnant gypsies under the age of 18, even users of substances or drugs that act in the central nervous system or even generally pregnant women who did not have a smooth and normal pregnancy. For all these reasons, collecting a sample for the survey was extremely difficult.

Our aim is for this research to continue and expand with subsequent studies. If our research manages to consolidate music in clinical practice, we expect a reduction in Caesarean sections due to false nonreassuring cardiotocographys. The music enables us to distinguish false non-reassuring cardiotocographys from pathological cardiotocographys resulting in, the reduction of Caesarean sections. It would be equally important to do additional research that would compare the duration of the first stage of childbirth respectively of the group music and of the control group (as was done in our previous pilot study in 2015), in order to prove that the Music Group would have a shorter first stage of childbirth, which would be very important for both pregnant women and health professionals. Moreover, research could be carried out where Music will reduce the examination time in large obstetric ultrasounds such as the Level 2 Ultrasound (or Anomaly Scan). But no matter how many investigations become, our goal it's one. Music, must gain solid foundations and establish itself not only in maternity hospitals but also in all healthcare facilities because it is a drug without side effects and with a zero cost.

\section{Conflicts of interest}

None to declare

\section{Sources of Funding}

None

\section{References}

1. Sbrollini A, Agostinelli A, Marcantoni I. eCTG: an automatic procedure to extract digital cardioto- 
cographic signals from digital images. Comput Methods Programs Biomed. 2018;156:133-139.

2. National German Guideline (S1). Use of CTG During Pregnancy and Labour, AWMF Registry No. 015/036. Geburtsh Frauenheilk 2014; 74: 721-732

3. MIDIRS and the NHS centre for reviews and dissemination. Fetal Heart Rate Monitoring in Labour. Informed choice for professionals leaflet. 2003. Retrieved May 31st 2020.

4. NICE. Inherited Clinical Guideline C. "The use and interpretation of cardiotocography in intrapartum fetal surveillance". 2001. http://ctgutbildning.se/ images/Referenser/NICE-guidelines-FHR-monitoring-2001.pdf. Retrieved May 31st 2020.

5. Bernardi L, Porta C, et al. Dynamic interactions between musical, cardiovascular and cerbral rhythms in humans. Circulation. 2009;119:3171-80.

6. Cervellin, G. Lippi, G. From music-beat to heartbeat: A journey in the complex interactions between music, brain and heart. Eur J Intern Med. 2011;22:371-4.

7. García-Casares N, Martín-Colom J. García-Arnés J. Music Therapy in Parkinson's Disease. J Am Med Dir Assoc. 2018;19:1054-62.

8. Gómez Gallego M, Gómez García J. Music Therapy and Alzheimer's Disease: Cognitive, Psychological, and Behavioural Effects. Neurología 2017;32:300-8.

9. Moumdjian L, Moens B, Maes P. Walking to Music and Metronome at Various Tempi in Persons With Multiple Sclerosis: A Basis for Rehabilitation. Neurorehabil Neural Repair. 2019;33:464-75.

10. Milona G, Dimitrova A, Iliadou M. Study of the effect of music on the mother and the fetus in relation to the mother's psychology and the cardiotocography in pregnancy and childbirth. 2014

11. Packyanathan JS, Lakshmanan R. Effect of Music therapy on anxiety levels on patient undergoing dental extractions. J Family Med Prim Care. 2019; 8: 3854-60.
12. Yamashita K, Kibe T, et al. The effects of music listening during extraction of the impacted mandibular third molar on the autonomic nervous system and psychological state. J Oral Maxillofac Surg. 2019;77:1153.e1-1153.e8.

13. Kishida M, Yamada Y, et al. Effectiveness of music therapy for alleviating pain during haemodialysis access cannulation for patients undergoing haemodialysis: a multi-facility, single-blind, randomised controlled trial. Trials. 2019;20:631.

14. Richard-Lalonde M, Gélinas $C$, et al. The effect of music on pain in the adult intensive care unit: $\mathrm{A}$ systematic review of randomized controlled trials. J Pain Symptom Manage. 2020;59:1304-1319.e6.

15. Jayakar Jai P., Alter David A. Music for Anxiety Reduction in Patients Undergoing Cardiac Catheterization: A Systematic Review and Meta-Analysis of Randomized Controlled Trials. Complement Ther Clin Pract. 2017;28:122-30.

16. Kafali, H., Derbent, A. et al. Effect of maternal anxiety and music on fetal movements and fetal heart rate patterns. J Matern Fetal Neonatal Med. 2011;24:461-4.

17. Gebusa, G., Zaleska, M. et al. The effect of music on the cardiac activity of a fetus in a cardiotocographic examination. Adv Clin Exp Med. 2018;27:615-21.

18. Pasternak Y., Miller N., Asali A. Does Music During Labor Affect Mode of Delivery in First Labor After Epidural Anesthesia? A Prospective Study. Arch Gynecol Obstet. 2019;300:1239-44

19. Garcia-Gonzalez J, Ventura-Miranda M, Requena - Mullor M. State-trait Anxiety Levels During Pregnancy and Fetal Parameters Following Intervention With Music Therapy. J Affect Disord. 2018;232:17-22.

20. García González J, Ventura Miranda M I, Requena Mullor M. Effects of Prenatal Music Stimulation on State/Trait Anxiety in Full-Term Pregnancy and Its Influence on Childbirth: A Randomized Controlled Trial. J Matern Fetal Neonatal Med. 
2018;31:1058-65.

21. Oh MO, Kim YJ, et al. Effect of Music Intervention on Maternal Anxiety and Fetal Heart Rate Pattern During Non-Stress Test. J Korean Acad Nurs. 2016;46:315-26.

22. Grażyna Gebuza, Marta Zaleska, Marzena Kaźmierczak et al. The Effect of Music on the Cardiac Activity of a Fetus in a Cardiotocographic Examination. Adv Clin Exp Med. 2018;2:615-21.
23. Hasan Kafali , Aysel Derbent, Esra Keskin et al. Effect of Maternal Anxiety and Music on Fetal Movements and Fetal Heart Rate Patterns. J Matern Fetal Neonatal Med. 2011;24:461-4.

Received 11-06-20

Revised 15-06-20

Accepted 22-06-20 